\title{
Signal and error assessment of GOCE-based high resolution gravity field models
}

DOI: https://doi.org/10.1515/jogs-2019-0008

Received November 19, 2018; accepted March 10, 2019

\begin{abstract}
The signal content and error level of recent GOCE-based high resolution gravity field models is assessed by means of signal degree variances and comparisons to independent GNSS-levelling geoid heights. The signal of the spherical harmonic series of these models is compared to the pre-GOCE EGM2008 model in order to identify the impact of GOCE data, of improved surface and altimetric gravity data and of modelling approaches. Results of the signal analysis show that in a global average roughly $80 \%$ of the differences are due to the inclusion of GOCE satellite information, while the remaining 20\% are contributed by improved surface data. Comparisons of the global models to GNSS-levelling derived geoid heights demonstrate that a $1 \mathrm{~cm}$ geoid from the global model is feasible, if there is a high quality terrestrial gravity data set available. For areas with less good coverage an accuracy of several centimetres to a decimetre is feasible taking into account that GOCE provides now the geoid with a centimetre accuracy at spatial scales of 80 to $100 \mathrm{~km}$. Comparisons with GNSS-levelling geoid heights also are a good tool to investigate possible systematic errors in the global models, in the spirit levelling and in the GNSS height observations. By means of geoid height differences and geoid slope differences one can draw conclusions for each regional data set separately. These conclusions need to be considered for a refined analysis e.g. to eliminate suspicious GNSSlevelling data, to improve the global modelling by using full variance-covariance matrices and by consistently weighting the various data sources used for high resolution gravity field models. The paper describes the applied procedures, shows results for these geoid height and geoid slope differences for some regional data sets and draws conclusions about possible error sources and future work to be done in this context.
\end{abstract}

\footnotetext{
T. Gruber: Institute of Astronomical and Physical Geodesy Technical University of Munich, Arcisstrasse 21, 80333 Munich, Germany, E-mail: Thomas.Gruber@tum.de

M. Willberg: Institute of Astronomical and Physical Geodesy Technical University of Munich, Arcisstrasse 21, 80333 Munich, Germany
}

Keywords: Global gravity field model, GNSS-levelling, GOCE

\section{Introduction}

Since the completion of the GOCE mission and the availability of the release 5 GOCE-based satellite-only gravity field models (Brockmann et al. 2014; Bruinsma et al. 2013), a number of high resolution combined global gravity field models has been published through the International Centre for Global Earth Models (ICGEM) (Barthelmes and Köhler 2016). These models all have in common that they combine latest satellite information from GOCE, GRACE and other satellites with terrestrial, airborne and altimetric data in order to provide the global Earth gravity field with highest possible resolution, considering the spatial resolution of available surface data. In general, one can regard them as the static (mean) part of the Earth gravity field without a specific time stamp, as they combine data and observations obtained over decades. In contrast, there are available several monthly (and shorter period) time series computed from GRACE data providing the temporal evolution of the Earth gravity field and subsequently mass transport in the Earth system. The time variable gravity field will not be considered in this study. Among a few others, the precursor of the new generation high resolution static gravity field models is the EGM2008 model (Pavlis et al. 2012), which, until nowadays, represents the standard model used worldwide for many applications. EGM2008 is based on a few years of GRACE observations, information from a variety of satellites and terrestrial and altimetric gravity data available at that time.

High resolution static gravity field models are used for a wide range of applications in geodesy, solid Earth sciences and oceanography. As a few examples of many, one could mention geoid based height determination in areas with less good ground data coverage (Li et al. 2015), analysis of the structure of the Earth's crust (Evariste et al. 2014) and determination of the mean ocean dynamic topography (Andersen and Knudsen 2009; Bingham et al. 2008). With the newly published high resolution gravity field models one can expect significant improvements with 
respect to EGM2008, specifically from the inclusion of the GOCE gravity gradiometer data, from the analysis of the extended GRACE observation time series and partially also from newly observed terrestrial and airborne gravity data as well as from improved altimetric gravity. In order to identify the impact of the new information, but also to identify possible improvements due to better modelling approaches, these high resolution models need to be assessed in terms of their signal content and their absolute error by comparing them to the EGM2008 model and to independent geoid information. For the latter only geoid heights over land observed by the so-called GNSS-levelling technique can be applied, because they are not used as observation in high resolution models. These GNSS-levelling geoid heights are determined pointwise by subtracting the orthometric or normal height determined by spirit levelling and gravimetry from the ellipsoidal height observed by GNSS (Heiskanen and Moritz 1967, Chapter 4). These comparisons applied to a number of newly released high resolution gravity field models represent the main focus of this paper, which is structured as follows. In chapter 2 the comparison methods are shortly summarized and critically assessed. Chapter 3 provides a detailed description of the data sets used for the study, while in chapters 4 and 5 the results of the comparisons are presented and interpreted. Chapter 6 finally discusses the obtained results and derives some conclusions for future work.

\section{Methods and Procedures}

The signal content of global gravity field models, which are available as spherical harmonic series, best is analysed by means of signal degree variances (Heiskanen and Moritz 1967, Chapter 7-3). They are reflecting the global average signal per degree of a spherical harmonic series corresponding to a specific wavelength, which can be approximated by half of the circumference of the Earth divided by the degree $\mathrm{N}(20.000 \mathrm{~km} / \mathrm{N})$. As the main goal of this study is to assess the impact of new satellite and surface information with respect to the pre-knowledge, which is represented by the EGM2008 model, signal differences to EGM2008 model are computed and represented in terms of difference signal degree variances. These differences show at which wavelength the models differ more or less and where the new information enters most. Finally, in order to identify the average contribution of new information with respect to EGM2008, the difference signal variances can be accumulated up to degree $\mathrm{n}$ with the cumulative sum determining the effect of new information up to a specific special resolution.

The absolute accuracy is assessed by comparison of model derived geoid heights (or height anomalies) to independent geoid heights at GNSS-levelling stations. The procedure follows to a large extent the method as described in Gruber et al. (2011). As it is quite important to interpret these differences in a correct way the basic processing steps are repeated again. In addition to the standard comparisons shown in Gruber et al. (2011), we introduce some new statistical analyses, which are based on geoid slopes between all GNSS-levelling points of a regional data set.

Step 1: Spherical harmonic coefficients need to be transformed to the constants of the reference ellipsoid of the GNSS observations. Normal gravity field and geometric reference ellipsoid (semi major axis) need to be consistent. In addition, the spherical harmonic series needs to be transformed to the permanent tide system of the GNSS levelling points (if required) (Gruber et al. 2014).

Step 2: Computation of geocentric coordinates for the GNSS-levelling points from ellipsoidal coordinates observed by GNSS and solution of the spherical harmonic series of the gravity field model at this location on the Earth surface up to degree $\mathrm{N}$ for height anomalies (Gruber et al. 2014).

Step 3: Estimation of the omission error, i.e. the gravity field signal not represented by the spherical harmonic series as it is used in step 2 (up to degree N). The main component is computed from an existing high resolution gravity field model (e.g. EGM2008 up to degree 2190). In case $\mathrm{N}$ is below the maximum degree of this high resolution model, the omission error is computed from degree $\mathrm{N}+1$ to the full resolution of the model (e.g. degree 2190 in case of EGM2008). In addition, the remaining omission error above the maximum degree is computed from a topographic gravity field model estimating the gravity signal from the residual topography above the maximum resolution of the high resolution gravity field model. Here we use the ERTM2160 model (Hirt et al. 2014). This model represents the short scale Earth gravity field above degree and order 2160 computed by forward modelling of an improved Shuttle Radar Topography Mission (SRTM) topography model and spectral filtering. It shall be noted that the spectral content of the high resolution gravity field models is limited to degree and order 2160 . The coefficients between degree 2160 and 2190 are artificially generated from the conversion of ellipsoidal harmonics into the spherical domain (Pavlis et al. 2012).

Step 4: In case orthometric height at GNSS-levelling points are available an additional correction needs to be applied to the height anomalies computed from the global 
model in order to get geoid heights. This term, also called geoid-quasi geoid separation, is computed according to Rapp (1997) and is strongly dependent on the height of the GNSS-levelling point. As an additional error source the topographic bias affects the results obtained in high mountain areas in case orthometric heights are used (Sjöberg 2018). As the density of the topography and its variations between the Earth surface and the geoid is not known the geoid-quasi geoid separation term as well as the topographic bias degrade the quality of the reference geoid heights in mountainous areas. This needs to be considered when the differences between observed, and from global models computed, geoid heights are analysed.

Step 5: These height anomalies or geoid heights then can be directly compared to the corresponding results at the GNSS-levelling points, which are computed by subtracting the orthometric height or the normal height from the ellipsoidal GNSS height. These differences can be analysed per regional data set either by regarding the difference maps or by statistical analyses.

Step 6: In many cases the differences for a regional data set exhibit systematics in terms of biases or tilts. Biases are caused by different definitions of national height systems, but can be neglected for the validation of global gravity field models as long as the bias for a regional data set is constant. Tilts, on the other hand, can be caused by a deviation of the national height reference surface from an equipotential surface or by systematic errors in the spirit levelling network (Wang et al. 2012). For validation of global gravity field models these effects need to be accounted for by disregarding the mean difference (i.e. the bias) and by computing a planar correction surface which is applied to the height anomalies or geoid heights, respectively.

Step 7: After applying the correction surface the root mean square (RMS) of the differences between the model geoid and the observed GNSS-levelling geoid is computed per region as a validation result. The RMS value is a quality criterion which tells how good the global model fits to the observed geoid or height anomalies.

Step 8: These geoid differences per GNSS-levelling point can be further analysed by computing differences of geoid differences between all points of a regional network, which we call geoid slope differences in the following. These geoid slope differences can be analysed with respect to the distance or the height difference between two points or with respect to the orientation of the geoid slope difference. By statistical analysis of geoid slope differences for distance, height difference or orientation class one is able to obtain a more detailed insight into possible problems either in the global model or in the GNSS-levelling geoid data. Finally, also combination of classes can be regarded providing information about possible correlations of geoid slope differences.

The procedures as described above are applied to a number of available GNSS-levelling data sets, which are introduced in the following chapter.

\section{Data Sets}

In this study a quality assessment of high resolution gravity field models incorporating at least a multi-year data set of the GRACE mission and the complete GOCE data set is performed. These models are compared against the EGM2008 model (Pavlis et al. 2012), which is based on a limited GRACE data set but no GOCE information. Table 1 provides an overview about the high resolution models, which are used in this study.

Each model is discussed in more detail, specifically regarding the information which has been used to compute the model, as this is important to correctly interpret the validation results.

EGM2008: This model, until nowadays, represents the standard for many applications. It doesn't contain data from the GOCE mission, but an early GRACE mean gravity field based on 4 years of data. The model was computed by a combination of full normal equations up to degree and order 359 and a reduced block-diagonal structure for the ellipsoidal spectrum up to degree 2159. After transformation from the ellipsoidal into the spherical spectrum the maximum resolution is degree 2190 and order 2159.

EIGEN6-C4: The model is based on 10 years of GRACE data, the complete GOCE mission data set and about 25 years of LAGEOS observations. Over the oceans EIGEN6C4 includes a recent version of DTU altimetric gravity anomalies, while over land it applies mostly data computed from the EGM2008 model. The model was computed in the spherical domain with a full normal equation system up to degree 370 and a reduced block-diagonal normal equation system up to degree 2190 . When comparing this model with GNSS-levelling geoid heights it has to be considered that only the satellite part was significantly improved, while over land the information is mainly identical to EGM2008. The updated altimetric gravity data mostly provide better information in the coastal regions, while in the open ocean it is very similar to the EGM2008 data set.

GECO: The goal of this model was to insert better satellite information from GOCE into EGM2008. For this purpose, a pure GOCE model containing the full mission data set was spectrally combined by with the EGM2008 model. 
All terrestrial and altimetric data are therefore identical to EGM2008.

GOCE-OGMOC: This model is a combination between the XGM2016 (Pail et al. 2018) and the EIGEN6-C4 model (Förste et al. 2014). It is identical to XGM2016 up to degree 619, between degree 620 and 719 both models are spectrally combined applying a weighting function and from degree 720 to 2190 it is identical to EIGEN6-C4. Both models include identical altimetric gravity anomalies, while over land an improved 15’x15' block-mean data set of gravity anomalies provided by the National GeospatialIntelligence Agency (NGA) was used for XGM2016. This data set was extracted from the NGA database of terrestrial and altimetric gravity data and incorporates in some regions newly observed gravity data from airborne campaigns. In addition, improved data editing and preparation were applied to the data before the calculation of the block-mean values. In the spectral range from degree 620 to 2190 , according to the weights, terrestrial information (over land) from the EGM2008 model is introduced over land. As satellite information a GRACE/GOCE combination model was used, which includes the complete GOCE mission data set and most of the GRACE data [GOCO05S (Mayer-Gürr and the GOCO Team 2015)].

SGG-UGM-1: This model combines a GOCE satellite model with terrestrial and altimetric data computed from the EGM2008 model. The model is computed by an adjustment approach applying full normal equations up to degree and order 220 and a reduced block-diagonal system up to degree 2159. All terrestrial and altimetric data are therefore identical to EGM2008.

PGM2017: The data used for this model are not completely known, but it can be assumed that similar gravity data as for the XGM2016 is applied. Only for some regions updated data were introduced. For this model the terrestrial and altimetric data are not spectrally limited to degree 719, but block-mean values with 5'x5' spatial resolution are used. For the satellite part it can be assumed that the GOCO05S model is applied. Therefore, the model up to degree 620 is very similar to the GOCE-OGMOC solution, while for higher degrees improved information is available. The estimation method applied for this model is unknown.

Apart from the global models various sets of GNSSlevelling data are used, which are summarized in Table 2. In general, these data sets are provided in the form of orthometric heights from the spirit levelling, which are subtracted from the ellipsoidal heights from GNSS in order to compute geoid heights. Only for Germany and the unified European data set normal heights and height anomalies are available. The number of stations and subsequently their density in each region vary significantly (refer to Table 2, column 2).

\section{Signal Assessment}

For an initial assessment of the signal content of the global models geoid height differences between the high resolution models under investigation and EGM2008 are computed and displayed in terms degree variances (square root) (i.e. global mean of model differences in the spectral domain). Figure 1 shows these differences from which one is able to identify the influence of the new satellite information from GOCE and GRACE and the newly included terrestrial and altimetric gravity data.

The largest differences occur for degree 60 to 200, which are dominated by the contribution of the GOCE data (see Fig. 1, bottom). All models under investigation now contain GOCE information and therefore the differences to EGM2008 (without GOCE) are at similar level. For the long wavelengths below degree 60 one can identify the value of GRACE information, which was included already in EGM2008, but not in the GECO and SGG-UGM-1 models. For this reason, both models exhibit larger differences in this frequency range. Finally, for the high frequencies above degree 200 the contribution of new terrestrial and altimetric gravity data can be identified (Fig. 1, top). The largest differences to EGM2008 exist for PGM2017, which includes a new set of 5'x5' surface data. All other models use prior information from EGM2008 starting at a specific degree. This becomes visible by the strong drops in the degree variance difference curves. For the GOCE-OGMOC model a new terrestrial/altimetric data set with $15^{\prime} \times 15^{\prime}$ resolution was used, which corresponds to degree 719 while for other models EGM2008 information entered already at degree 360 or smaller (compare Table 1).

In order to quantify how much information in global average is from the GOCE data and how much from new terrestrial and altimetric information the signal difference degree variances are accumulated and shown in Fig. 2 in terms of the square root of cumulative signal degree variance differences as geoid heights. As one clearly can identify, most of the differences to EGM2008 are in the spectral range up to degree 200, which is caused by the GOCE data (about $80 \%$ of the total difference at degree 2190). The remaining differences mostly are between degree 200 and 500 (about $20 \%$ of total) and only marginal contributions are in the spectral range above degree 500 . This is well visible by the slope of the curves. The steeper the slope the more contribution from new data up to this de- 
Table 1. Overview of high resolution global gravity field models. Degree/Order: maximum resolution of the model in the spectral domain (sph. = spherical domain, ell. = ellipsoidal domain); Satellite Data: satellite data applied in the model; Surface Data: altimetric and land data sets used in the model; Method: combination method, full = usage of full normal equations, BD = block-diagonal normal equations, Spectral combination $=$ combination of models.

\begin{tabular}{|c|c|c|c|c|c|}
\hline Model & Degree/ Order & Satellite Data & Surface Data & Method & Ref. \\
\hline EGM2008 & $\begin{array}{l}2159 \text { ell. } \\
2190 \mathrm{sph} .\end{array}$ & ITG-GRACE03S & $\begin{array}{l}\text { DNSC07 Altimetry } \\
\text { SS v18.1 Altimetry } \\
\text { NGA08 Land }\end{array}$ & $\begin{array}{l}\text { d/o } 359 \text { full } \\
\text { d/o } 2159 \text { BD }\end{array}$ & $\begin{array}{c}\text { (Pavlis et al. } \\
\text { 2012) }\end{array}$ \\
\hline EIGEN6-C4 & $2190 \mathrm{sph}$. & $\begin{array}{l}\text { GRACE-GRGS (10y) } \\
\text { GOCE-DIR5 } \\
\text { LAGEOS }\end{array}$ & $\begin{array}{l}\text { DTU10 Altimetry } \\
\text { EGM2008 Land }\end{array}$ & $\begin{array}{l}\text { d/o } 370 \text { full } \\
\text { d/o } 2190 \text { BD }\end{array}$ & $\begin{array}{c}\text { (Förste et al. } \\
\text { 2014) }\end{array}$ \\
\hline GECO & $2190 \mathrm{sph}$. & GOCE-TIM5 & $\begin{array}{l}\text { EGM2008 Land EGM2008 } \\
\text { Ocean }\end{array}$ & $\begin{array}{l}\text { Spectral } \\
\text { combination on } \\
\text { geoid }\end{array}$ & $\begin{array}{l}\text { (Gilardoni et } \\
\text { al. 2016) }\end{array}$ \\
\hline GOCE-OGMOC & $2190 \mathrm{sph}$. & GoCo05S & $\begin{array}{l}\text { DTU13 Altimetry } \\
\text { NGA16 Land (15') }\end{array}$ & $\begin{array}{l}\text { Spectral } \\
\text { combination } \\
\text { XGM2016 (Pail et al. } \\
\text { 2018), EIGEN6-C4 } \\
\text { (Förste et al. 2014) }\end{array}$ & $\begin{array}{c}\text { (Gruber and } \\
\text { Fecher 2018) }\end{array}$ \\
\hline SGG-UGM-1 & $2159 \mathrm{sph}$. & GOCE & $\begin{array}{l}\text { EGM2008 Land } \\
\text { EGM2008 Ocean }\end{array}$ & $\begin{array}{l}\text { d/o } 220 \text { full } \\
\text { d/o } 2159 \text { BD }\end{array}$ & (Liang 2018) \\
\hline PGM2017 & $\begin{array}{l}2159 \text { ell. } \\
2190 \mathrm{sph} .\end{array}$ & GoC005S & $\begin{array}{l}\text { DTU17 Altimetry } \\
\text { SS2017 Altimetry } \\
\text { NGA17 Land (5') }\end{array}$ & unknown & (NGA 2018) \\
\hline
\end{tabular}

Table 2. Overview of GNSS-levelling datasets used for the study

\begin{tabular}{lcll}
\hline Region & $\begin{array}{c}\text { No. } \\
\text { Points }\end{array}$ & & Reference \\
\hline Australia & 197 & Geoscience Australia, 2003 & No. \\
Brazil & 683 & $\begin{array}{l}\text { Brazilian Institute of Geography and Statistics - IBGE, Directorate of Geosciences - } \\
\text { DGC, Coordination of Geodesy - CGED, 2012 }\end{array}$ & G02 \\
Canada & 579 & National Resources of Canada (NRCan), via US NGS, 2012 & G03 \\
Europe various & 2576 & National Resources of Canada (NRCan), 2007 & G04 \\
countries, EUREF EUVN & 1233 & Bundesamt für Kartographie und Geodäsie, Frankfurt/Main, 2007 & G05 \\
Germany & 675 & Bundesamt für Kartographie und Geodäsie, Frankfurt/Main, 2003 & G06 \\
Great Britain & 470 & ○ GeoBasis-DE / Geobasis NRW, 2018 & G07 \\
Greece (mainland) & 177 & UK Ordnance Survey, 2011 & G08 \\
Japan & 1542 & Aristotle University of Thessaloniki, 2016 & G09 \\
Mexico & 837 & Japanese Geographical Survey Institute, 2003 & G10 \\
Saudi Arabia & 744 & Instituto Nacional de Estadística y Geografía (México) via US NGS, 2012 & G11 \\
USA & 382 & King Abdulaziz City for Science and Technology KACST, 2012 & G12 \\
\hline
\end{tabular}

gree is present. When the line becomes horizontal, from this degree onwards, hardly any additional contribution with respect to EGM2008 is in the investigated high resolution gravity field model.

In summary, one can state that the inclusion of GOCE data significantly changes the high resolution gravity field models in the spectral range up to degree and order 200 with respect to EGM2008 and that new terrestrial and altimetric data mostly contribute to differences between degree 200 and 500. The PGM2017 model, which makes use of GOCE data and an updated terrestrial/altimetric gravity data set exhibits the largest differences immediately followed by the GOCE-OGMOC model, which uses new surface gravity data with reduced spatial resolution (15'x15'). The remaining models rely on information taken from EGM2008 above degree 360. It shall be noted that larger differences to EGM2008 do not necessarily mean that the new high resolution model is better than previous ones. This needs to be assessed by comparisons to independent 

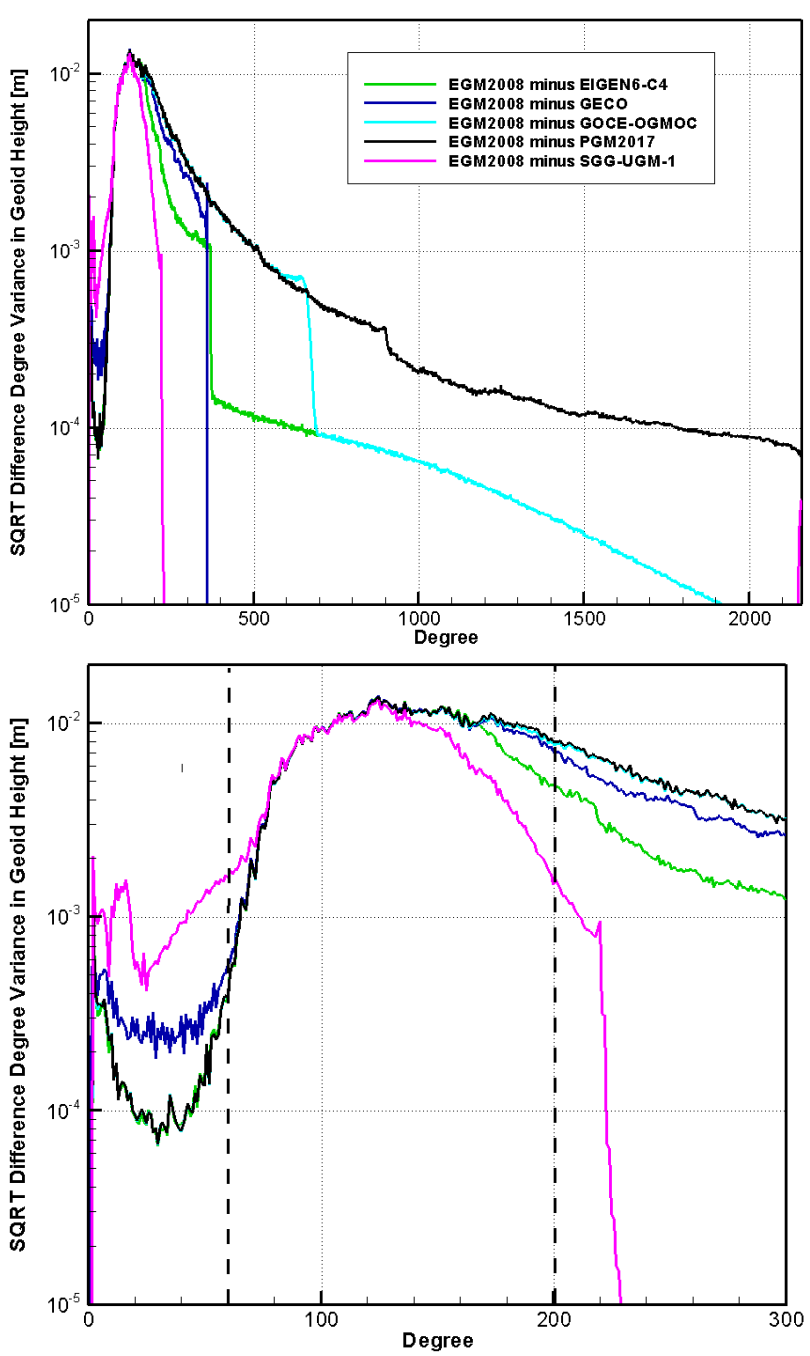

Fig. 1. Geoid height differences in terms of square root of difference signal degree variances between EGM2008 and high resolution gravity field models. Top: for the full resolution up to degree 2190; Bottom: Zoom in up to degree 300 (dashed lines mark the degrees 60 and 200).

gravity field information (e.g. from GNSS-levelling) as it is shown in the next chapter.

\section{Error Assessment}

The quality of the high resolution gravity field models is assessed by comparisons to independently observed geoid heights at GNSS-levelling stations. The procedure is described in detail in chapter 2 (steps 1 to 6 ), while the GNSSlevelling data sets are summarized in Table 2. First, for one regional example, the raw differences are computed, the estimated planar correction surface is estimated and residual differences after applying the correction surfaces

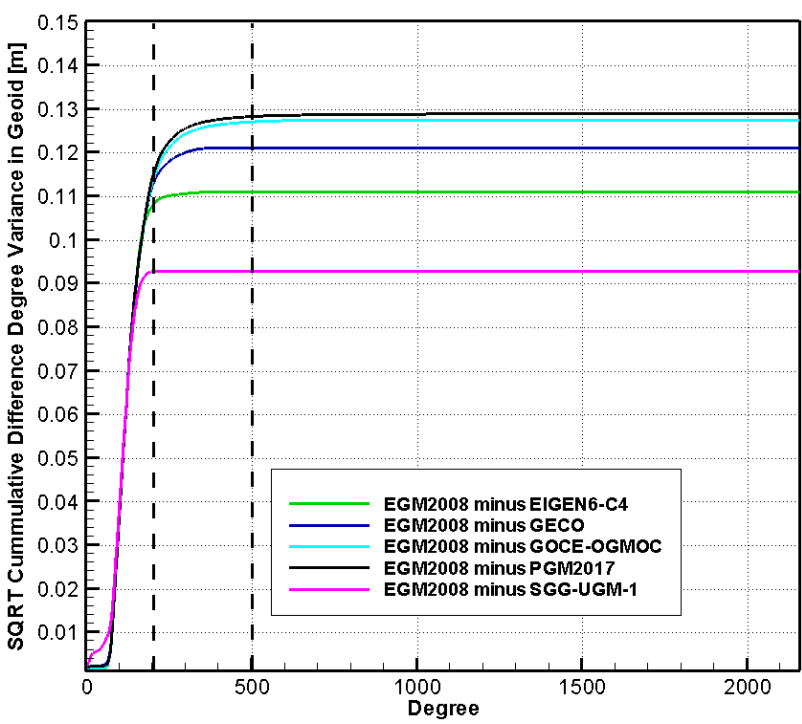

Fig. 2. Geoid height differences between EGM2008 and high resolution models under investigation as square root of cumulative signal degree variance differences in terms of geoid heights (dashed lines mark the degrees 200 and 500).

are computed. Here we use the German data set from 2018 (G07) (refer to Table 2) as well as the EGM2008 and PGM2017 high resolution models (refer to Table 1). Figure 3 shows the results of the pointwise geoid differences between the models for the full resolution up to degree 2190 (taking into account the remaining omission error from the topographic gravity model) and the independent geoid heights observed at the GNSS-levelling stations before and after applying the planar correction surface and the correction per point.

For the German data set (G07) (Fig. 3) it can be identified that for PGM2017 the raw differences vary slightly more than for EGM2008. After applying the planar correction some differences between the two models can be observed. In particular, locations showing larger differences have changed and differences for PGM2017 in general seem to be slightly larger. Regarding the planar correction surface they look similar for both models, but the tilt of the planar correction surface in North-South direction (approximately) is slightly larger for PGM2017 than for EGM2008.

From this kind of figures it is hardly possible to determine which model performs better. Therefore, the RMS of geoid differences (after subtracting the planar correction surface) is computed for all points of a regional data set using the full resolution of the high resolution gravity field models and applying the remaining omission error from the residual topographic gravity field (refer to chapter 2 

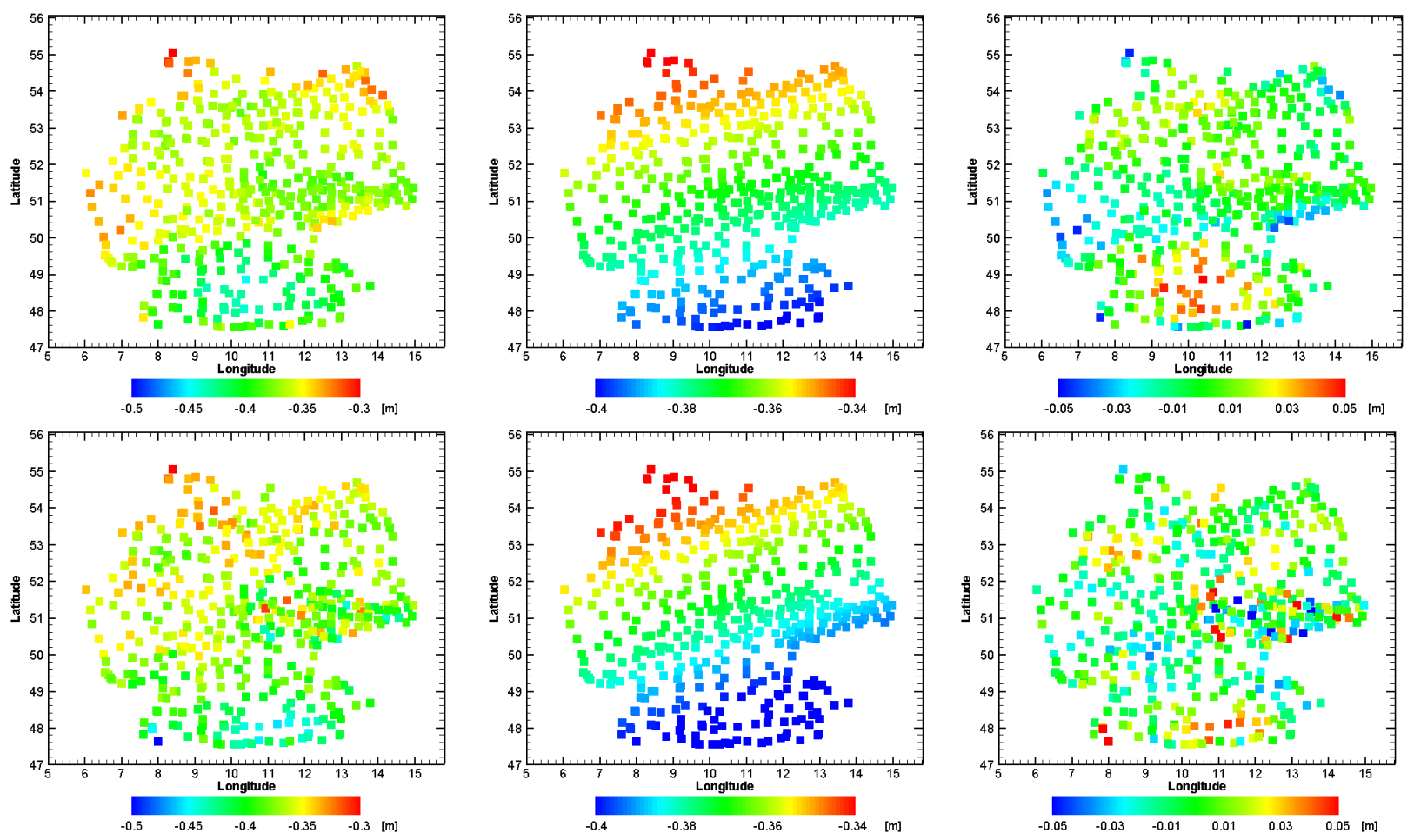

Fig. 3. Differences of EGM2008 and PGM2017 height anomalies to GNSS-levelling height anomalies in Germany (data set G07) for full model resolution (degree 2190). All units in [m]. Top row: EGM2008 model; Bottom row: PGM2017 model; Left column: Raw differences without applying planar correction surface; Middle column: Correction surface at GNSS-levelling points; Right column: Differences after applying the correction.

step 3). Table 3 shows the results for these comparisons.

Table 3. RMS of geoid differences per regional data set in $[\mathrm{cm}]$. Global models are computed with full resolution. A planar correction surface is applied for each combination. First column: Region and country code (refer to Table 2). For G05, subsets for some European countries (see country code) and for G13, subsets for some US states (see state code) are considered. The colour code indicates the ranking per data set: Green = best, Yellow = closer to best; Orange $=$ closer to worst; Red $=$ worst.

\begin{tabular}{|c|c|c|c|c|c|c|}
\hline $\begin{array}{l}\text { GNSS-levelling } \\
\text { Dataset }\end{array}$ & EGM2008 & EIGENG-C4 & GECO & GOCE-OGMOC & SGG-UGM-1 & PGM2017 \\
\hline G01(AUS) & 17. & 17.5 & 17.9 & 17.6 & 17.8 & 17.6 \\
\hline G02 (BR) & 33.7 & 27.6 & 28.3 & 26.6 & 28.0 & 26.6 \\
\hline G03 (CDN) & 8.6 & 8.2 & 8.3 & 8.1 & 8.4 & 8.2 \\
\hline G04 (CDN) & 8.1 & 7.7 & 7.6 & 7.6 & 8.0 & 7.6 \\
\hline G05 (A) & 3.0 & 1.7 & 2.2 & 2.1 & 2.1 & 2.8 \\
\hline G05 (EST) & 4.7 & 3.8 & 4.4 & 2.2 & 4.4 & 2.2 \\
\hline G05 (FIN) & 6.9 & 5.0 & 5.9 & 4.8 & 6.1 & 4.7 \\
\hline $605(F)$ & 9.1 & 8.9 & 9.3 & 9.1 & 9.0 & 9.1 \\
\hline G05 (NL) & 1.7 & 2.0 & 2.1 & 1.9 & 1.4 & 1.6 \\
\hline G06 (D) & 2.0 & 2.1 & 2.4 & 2.4 & 2.4 & 2.7 \\
\hline G07 (D) & 1.8 & 1.5 & 1.9 & 1.7 & 2.0 & 2.1 \\
\hline G08 (GB) & 4.2 & 3.7 & 4.3 & 3.7 & 4.2 & 3.6 \\
\hline G09 (GR) & 13.9 & 12.4 & 13.4 & 13.0 & 13.1 & 13.1 \\
\hline G10 () & 7.4 & 6.5 & 6.8 & 6.3 & 6.3 & 6.3 \\
\hline G11 (MEX) & 30.1 & 29.9 & 29.2 & 28.3 & 29.2 & 28.6 \\
\hline G12 (KSA) & 51.6 & 50.2 & 50.1 & 48.5 & 50.0 & 48.8 \\
\hline G13 (USA) & 9.4 & 9.3 & 9.3 & 9.1 & 9.3 & 9.2 \\
\hline 613(AL) & 25.7 & 25.4 & 25.1 & 25.3 & 25.2 & 23.9 \\
\hline G13 (CA) & 8.3 & 8.2 & 8.3 & 8.1 & 7.9 & 8.3 \\
\hline G13 (CO) & 23.5 & 23.4 & 23.4 & 23.1 & 23.0 & 24.0 \\
\hline G13 (FL) & 4.5 & 5.0 & 4.1 & 3.6 & 4.0 & 3.7 \\
\hline G13(MN) & 3.4 & 3.7 & 3.7 & 3.5 & 3.7 & 3.5 \\
\hline G13 (NC) & 7.5 & 7.9 & 7.7 & 7.5 & 7.6 & 7.6 \\
\hline G13(SC) & 4.7 & 4.8 & 4.7 & 4.5 & 4.6 & 4.7 \\
\hline
\end{tabular}

The following intermediate conclusions can be drawn from Table 3:

- For the majority of regional differences EGM2008 performs worst. This is expected because in EGM2008 no GOCE data and no updated surface gravity data have been used.

- For the models which just merge GOCE information into the EGM2008 solution (GECO, SGG-UGM-1) the performance has been improved to some extent with respect to EGM2008. The SGG-UGM-1 model seems to outperform the GECO model for many regions.

- Models including GOCE and updated terrestrial and/or altimetric gravity data show best performance out of the three classes of gravity models and obviously the more updated terrestrial information is used the better the models fit to the independent GNSS-levelling geoid heights. The EIGEN6-C4 model indicates better performance than the models without updated surface data, while the GOCE-OGMOC and the PGM2017 models outperform EIGEN6-C4 because they make use of a new terrestrial land data set over large areas of the world. From the latter two models the GOCE-OGMOC model exhibits a slightly better performance, which is probably caused by the improved 
modelling approach using individual weighting and full variance-covariance matrices up to degree 719 .

- There are a few areas (Germany, Austria, California, Colorado), where the PGM2017 and the GOCE-OGMOC model perform worse than models based on EGM2008 surface information and GOCE. This is unexpected and cannot be explained at this point. However, the issue will be addressed in the following analyses.

- For some regions the level of geoid differences is quite high and in an absolute sense it seems that only marginal improvements can be identified between the models. As all errors contributing to these differences except for the global models are the same, any reduction of geoid differences can be attributed to the global models. Therefore, also in these cases the reduction of geoid differences RMS values seems to be meaningful and indicates which global model performs better.

In order to refine the analysis, the global models performance is evaluated for different truncation degrees. This means, that the omission error above the truncation degree is computed from a high resolution model (in this case the PGM2017 model is taken) and from the residual topographic gravity potential (refer to chapter 2). The impact of the truncation degree is investigated by applying different truncation degrees (degree and order 100, 120, 140, 160, $180,200,220,240,260,280,300,400,500,600,620,640$, $660,680,700,720,2160,2190)$. The reasons for selecting these truncation degrees are twofold. First, the influence of the satellite information from GOCE and GRACE shall be analysed (degree 100 to 300) and second, the impact of improved terrestrial information and the modelling approach shall be quantified (degree 400 to 2190). The sequence of truncation degrees between 620 and 720 was chosen in order to analyse the impact of full variance-covariance modelling versus a block-diagonal or diagonal approach (GOCE-OGMOC versus PGM2017). Finally, for each region and for each truncation degree the RMS of geoid differences between the model and the GNSS-levelling data is computed (in all cases the planar correction surface was applied). Figure 4 shows the RMS of these differences depending on the truncation degree for a selection of regional GNSS-levelling data sets.

Before drawing any conclusion from the results, Fig. 4 shall be explained shortly. In each sub-plot the black horizontal line represents the model used for computing the omission error. As PGM2017 was used in this case the RMS of the PGM2017 truncated model enhanced by the PGM2017 omission error always represents the full model. Therefore, for PGM2017 the RMS of the geoid differences is identical for all truncation degrees. All other models are a mix of the model under investigation (up to the degree of truncation) and PGM2017 for the omission error. Results can be interpreted by a relative comparison of the PGM2017 line with the results for the other models. In case a model line is below the straight line of PGM2017 this model for this degree of truncation fits better to the GNSS-levelling geoid heights and vice versa.

From a detailed analysis of the results shown in Fig. 4 the following conclusions can be drawn:

- One can observe that for all truncation degrees between 100 and 220, EGM2008 exhibits larger RMS values than all other models. This clearly indicates the impact of GOCE (and partially GRACE) satellite data, which were not used in EGM2008.

- For higher truncation degrees the impact of improved terrestrial and altimetric observations plays an increasing role. Models that contain solely terrestrial information from EGM2008 (GECO, SGG-UGM-1) in most cases exhibit larger RMS values than the models also incorporating new terrestrial and/or altimetric data. But, it also can be observed that most of the influence is from the improved GOCE information, while new surface data, specifically in areas where no terrestrial information was available in EGM2008, provide further improvements. This is nicely shown by the geoid differences in Brazil, where up to degree 220 for all models the RMS of the geoid differences is improved by roughly $6 \mathrm{~cm}$ with respect to EGM2008, while models with new terrestrial data (GOCE-OGMOC, PGM2017) reduce the RMS by about $7.5 \mathrm{~cm}$ for the full resolution. This implies that the new data contribute at a level of $1-2 \mathrm{~cm}$ RMS improvement in this case. Furthermore, this supports the findings in chapter 4 , where it was identified that about $80 \%$ of the overall improvement is due to the better satellite information, while about $20 \%$ is resulting from better terrestrial data.

- The previous conclusion is not fully supported by the results observed in Germany. Here the GOCE impact is visible as well, but it also is visible that for higher degrees all other models outperform the PGM2017 model, which is in opposite to all other regions shown in Fig. 4. The RMS of geoid differences for PGM2017 at its full resolution is between $20-30 \%$ larger than for all other models despite better satellite and updated terrestrial information. Specifically, it is visible, that with less impact of PGM2017 for computing the omission error (i.e. for increasing degrees of models based on EGM2008 information) the RMS of geoid height differences gets smaller. This can be very good observed from the GOCE-OGMOC model, which relies 

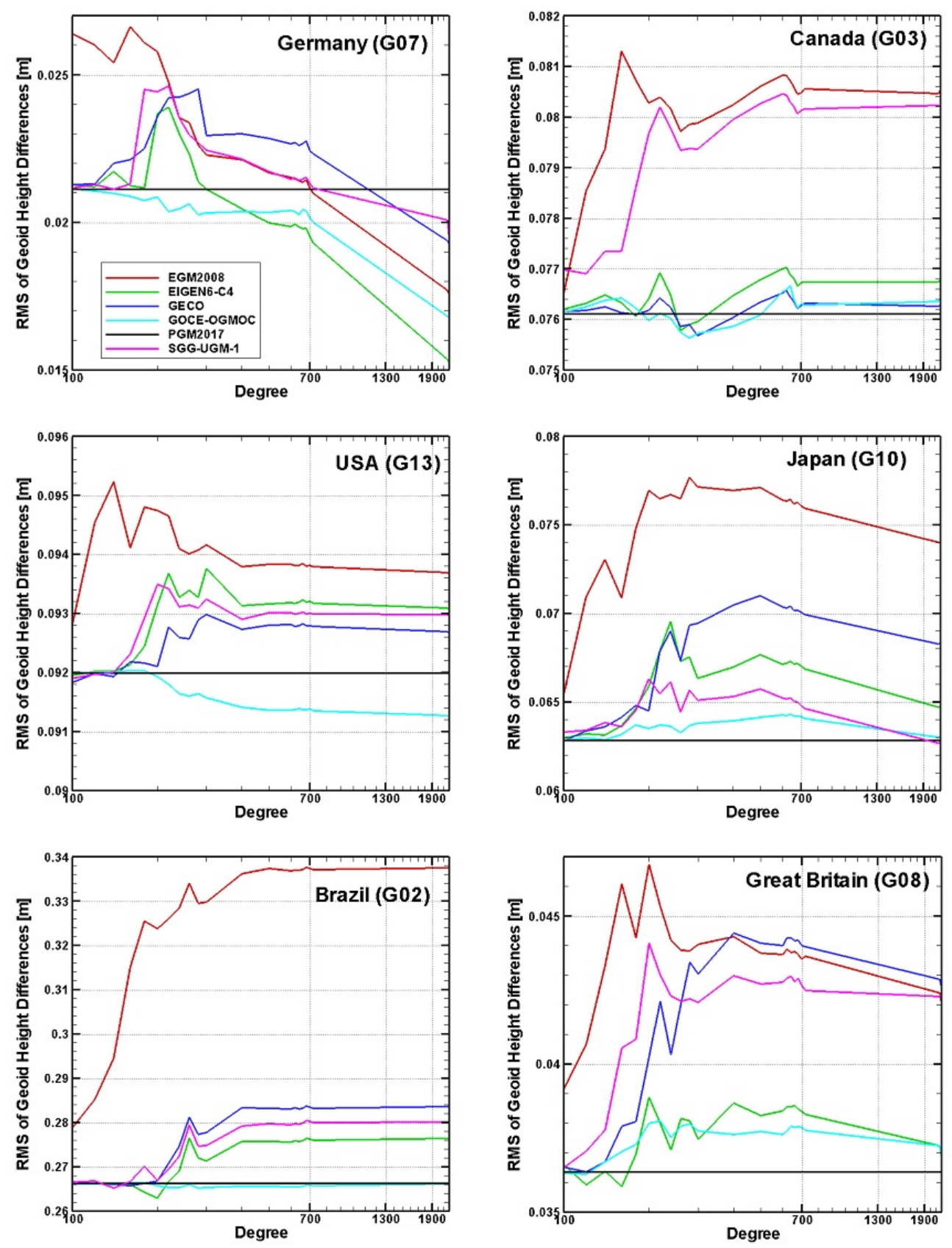

Fig. 4. RMS of geoid height differences (all in [m]) for selected regions and various truncation degrees of the global models. In all cases, the omission error was computed from the PGM2017 model and a residual topographic gravity field model. For all differences a planar correction surface was computed and subtracted.

up to degree 719 on the same input data as PGM2017, and which incorporates beyond degree 719 mostly EGM2008 derived information. As conclusion one can state that for the German GNSS-levelling data set high resolution gravity field models relying on EGM2008 information above the GOCE impact perform better than models relying on new terrestrial information in this area. The reason for this so far is unknown and needs to be identified by the PGM2017 development team. At the moment, it can only be speculated whether the terrestrial data set has some artefacts or the EGM2008 
model is specially adapted to the German surface gravity data set.

- Finally, it can be observed that for some regions (Germany, USA, Canada) the GOCE-OGMOC model slightly outperforms PGM2017 even if the majority of terrestrial and altimetric gravity data are identical (except that for GOCE-OGMOC only 15 'x15' resolution data were available while 5 ' $x 5^{\prime}$ data were available for PGM2017). This points towards the fact that the use of full normal equations with individual weighting of observations has some advantage compared to the other techniques (e.g. block-diagonal approach).

In order to further refine the error assessment of global high resolution gravity field models, investigations based on differences of geoid height slopes between all points of a regional GNSS-levelling network are performed. Geoid height slopes are determined from geoid height differences between two GNSS-levelling points, computed from the observed geoid heights and from the global models separately. Then differences of the two geoid slopes are computed. In the sequel we call them geoid slope differences. If this is done for all combinations of two points of a regional network, a large number of geoid slope differences is available, which then can be statistically analysed with respect to various parameters such as the distance, the height difference or the orientation between two points. Even a combination of two parameters is possible (refer to chapter 2, step 8). For this analysis only the full resolution of the global models (degree 2190) is considered. Figure 5 shows the results for selected areas.

The results of the geoid slope differences confirm the results of the geoid height differences (Table 3). All intermediate conclusions derived above also apply to the geoid slope differences. Regarding the geoid slope differences with respect to the distances between two points (Fig. 5, first row) one can identify some correlations of the RMS values with the point distance classes. In many cases larger RMS values are present for larger distances, what seems to be plausible as errors accumulate along the spirit levelling lines (even after subtracting a planar correction surface). Therefore, this behaviour can be addressed to levelling errors rather than to long wavelengths global gravity field model errors. For the interpretation of the statistical results it is also important to take into account the number of differences available for a distance class. This is shown by the black squares and the right scales in the figures. For larger distances and height differences usually significant less samples are available, which also can influence the RMS values. In general, larger RMS geoid slope differences are present for larger height differ- ences. This is plausible for two reasons. First, spirit levelling errors are increasing for larger height differences and second, possible model deficiencies when computing the residual topographic omission error might have some impact as well. This can't be explained at this point as one would need better knowledge about local situations related to point distribution and measurements. Regarding the results for the Brazilian data set it can be concluded that with the inclusion of the new satellite information from GOCE to the new high resolution models, the geoid slopes with respect to EGM2008 are significantly improved for all distance classes and for all height difference classes. As EGM2008 already contains some GRACE information the very long wavelengths of this model (corresponding to distances between GNSS-levelling points of $3000 \mathrm{~km}$ and more) are already determined with very good accuracy. The RMS of geoid slope differences with respect to the baseline orientation in contrast shows more peculiarities. Related to this analysis it needs to be mentioned that each GNSS-levelling point is combined with all others. For this reason, a symmetric pattern with an offset of $180^{\circ}$ orientation angle is caused. For all regions shown in Fig. 5 there can be identified larger RMS geoid slope differences for specific orientations. This is about $30^{\circ}\left(210^{\circ}\right)$ in Germany and $140^{\circ}\left(320^{\circ}\right)$ in Brazil. Basically one could suppose that larger geoid slope differences are related to the orientation of the planar correction surface, which has been applied (or its imperfect representation of possible levelling errors). In the case of Germany, the slope of the planar correction surface roughly has an orientation angle (with respect to North) of $330^{\circ}$ and is rotated by about $60^{\circ}$ with respect to the peak of the geoid slope differences. This might be an indicator that a planar correction surface is not sufficient to eliminate possible systematic errors. But a higher order correction surface can hardly be explained by systematic errors of spirit levelling or GNSS observations and neither by possible reference system differences between the geometric and gravimetric observations. The same holds for the high resolution global models, where systematic problems related to the orientation were not identified so far. More detailed analyses related to correction surfaces are required by analysing also other regional data sets, which is left for future work. For Germany it is also remarkable that the two models incorporating new surface gravity information and the complete GOCE and GRACE data (GOCE-OGMOC and PGM2017) exhibit only minor variations for the geoid slope differences, which points towards the conclusion that the levelling network in Germany has hardly any systematic problems after considering the planar correction surface (compare also to Fig. 3). 

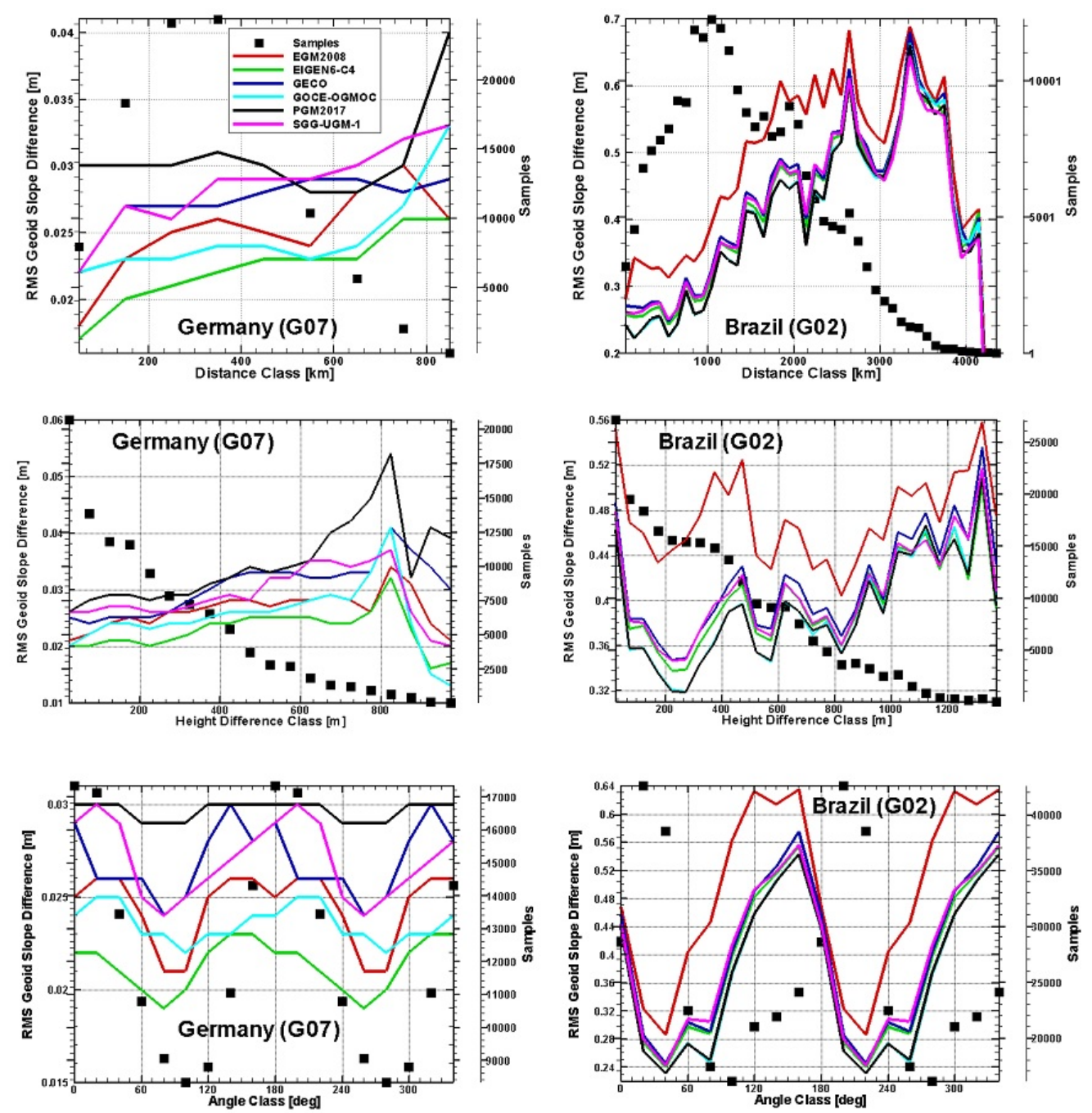

Fig. 5. RMS of geoid slope differences (all in [m]) between global models with full resolution (degree 2190) and GNSS-levelling points in Germany and Brazil. For all differences a planar correction surface was computed and subtracted from the geoid height differences. RMS of differences is shown for specific distance classes $(100 \mathrm{~km})$, for height difference classes $(50 \mathrm{~m})$ and for orientation classes (20 degrees) (left scale in each sub-figure). Black dots show the number of geoid slope differences per class (right scale in each sub-figure). Top row: RMS for distance classes; Middle row: RMS for height difference classes; Bottom row: RMS for orientation classes.

To further refine the analysis of geoid slope differences, in a final step, combinations of distance, height differences and orientation classes are investigated. For this purpose, geoid slope differences are sorted for combination of classes. For example, in case of a combination of distance and height difference classes, all geoid slope differences fulfilling both criteria are collected and statistically analysed (e.g. distance 100-200 km, height difference 200-250 m). For all possible combinations of two classes, i.e. distance and height difference, distance and orientation, height difference and orientation then RMS values of geoid slope differences are computed. The results are shown in Fig. 6 and 7 for two selected regions and two high resolution models. The regions were chosen such, that on one hand the region with the most precise GNSS-levelling data set was selected (Germany, data set G07) and on the other hand the region were the largest impact of new high resolution global models is visible (Brazil, data set G02). As global high resolution gravity field models according to Table 3 the worst model (EGM2008) and the best model (GOCE-OGMOC) were chosen. In general, the results show similar behaviour as for the single class analyses. By having a closer look to the results one can identify a few additional characteristics for the two regions. 
For Germany (Fig. 6) the following can be observed:

- The distance versus height difference analysis $\left(1^{\text {st }}\right.$ row) shows a more systematic pattern for GOCEOGMOC than for EGM2008. For example, one can identify a line from class $50 \mathrm{~km}$ distance (corresponding to distances in the range from 0 to $100 \mathrm{~km})$ and $225 \mathrm{~m}$ height difference (corresponding to height differences between 200 to $250 \mathrm{~m}$ ) to $650 \mathrm{~km}$ distance and $775 \mathrm{~m}$ height difference. Below this line the RMS values are at the $1-2 \mathrm{~cm}$ level while above this line RMS values are around $3 \mathrm{~cm}$. Such a line cannot be identified for the EGM2008 model. This means that the GOCE-OGMOC model has a very good performance for distances corresponding to the satellite information disregarding the height differences, but its performance is getting slightly worse for the shorter distances, which are dominated by the terrestrial data. Here EGM2008 performs better.

- The distance versus orientation analysis ( $2^{\text {nd }}$ row) shows the generally better consistency of the GOCEOGMOC model versus EGM2008 related to both parameters. For GOCE-OGMOC one can only identify a few classes with increased RMS values, which might point towards problematic GNSS-levelling geoid heights contributing to these classes. This tool might be usable to identify suspected GNSS-levelling points with larger uncertainty, which could be eliminated before performing these comparisons.

- From the height difference versus orientation analysis ( ${ }^{\text {rd }}$ row) no such clear conclusions can be derived. The patterns of larger RMS values are changing between both global models related to orientation, but for GOCE-OGMOC there is some improvement visible for smaller height differences independent of their orientation. This supports the observations already made above.

For Brazil (Fig. 7) in general the GOCE-OGMOC model performs significantly better than EGM2008, which was already identified in all previous analyses. Apart from that a few additional observations can be made from the combined class analysis.

- The distance versus height difference analysis (1st row) exhibits that for EGM2008 there are large differences for medium distance classes and low height difference classes. As this distance range is dominated by the satellite information this shows the impact of GOCE data and partially of improved surface gravity data.

- The distance versus orientation as well as height differences versus orientation results show significant differences for the two high resolution models. While for EGM2008 in both cases large RMS values of geoid slope differences are visible for most orientation angles for the GOCE-OGMOC geoid this reduces significantly. Independent whether distance classes or height difference classes are considered for GOCEOGMOC only two orientation angle classes exhibit large RMS values (between $330^{\circ}$ and $10^{\circ}$ orientation angle). As it is very unlikely that this is caused by the global model or the GNSS observations one can assume that the spirit levelling in some areas of the Brazilian network is significantly degraded and that there are inherent systematic errors in some levelling lines. In this case these comparisons are more useful to detect systematic problems in the spirit levelling rather than identifying systematic problems in the global models.

In summary one can state that this type of analyses delivers interesting insight into possible problems or correlations, which might be present in the global models, the GNSS observations, the spirit levelling network or the estimated correction surface. But, it is difficult to identify a single error sources just from these statistical analyses. Only by a joint analysis of all comparisons one might be able to identify the largest error source out of the four contributors. Nevertheless, as the various examples have shown, a joint interpretation of these results most likely enables the identification of GNSS-levelling points, which further-on can be excluded from the comparisons. This requires additional tools to locate such problematic points, which could be developed as a next analysis step.

\section{Summary and Conclusions}

The overall goal of the study was to evaluate the signal content and the errors of GOCE-based high resolution gravity field models. For this, geoid height differences in the spherical harmonic domain to the pre-GOCE gravity field model EGM2008 are computed and comparisons to a set of independently observed GNSS-levelling geoid heights are performed. In general, it can be concluded that with the inclusion of the GOCE data (and also with a longer time series of GRACE data) the quality of the global high resolution models has been significantly increased. In addition to that new or improved surface and altimetric gravity data further enhanced the quality, specifically in the higher frequencies of the models. Nevertheless, when comparing the results for the investigated models one can identify some 
RMS geoid slope differences EGM2008
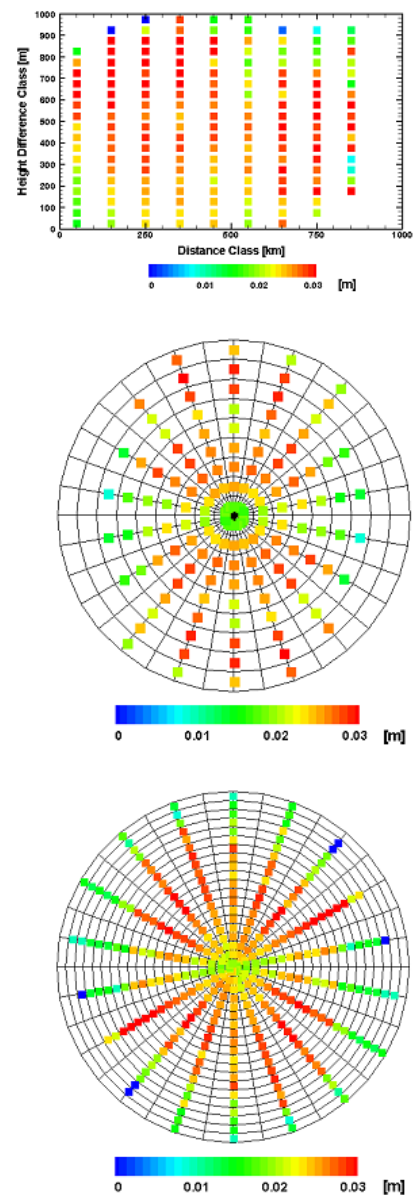

RMS geoid slope differences GOCE- Number of slope differences for class OGMOC
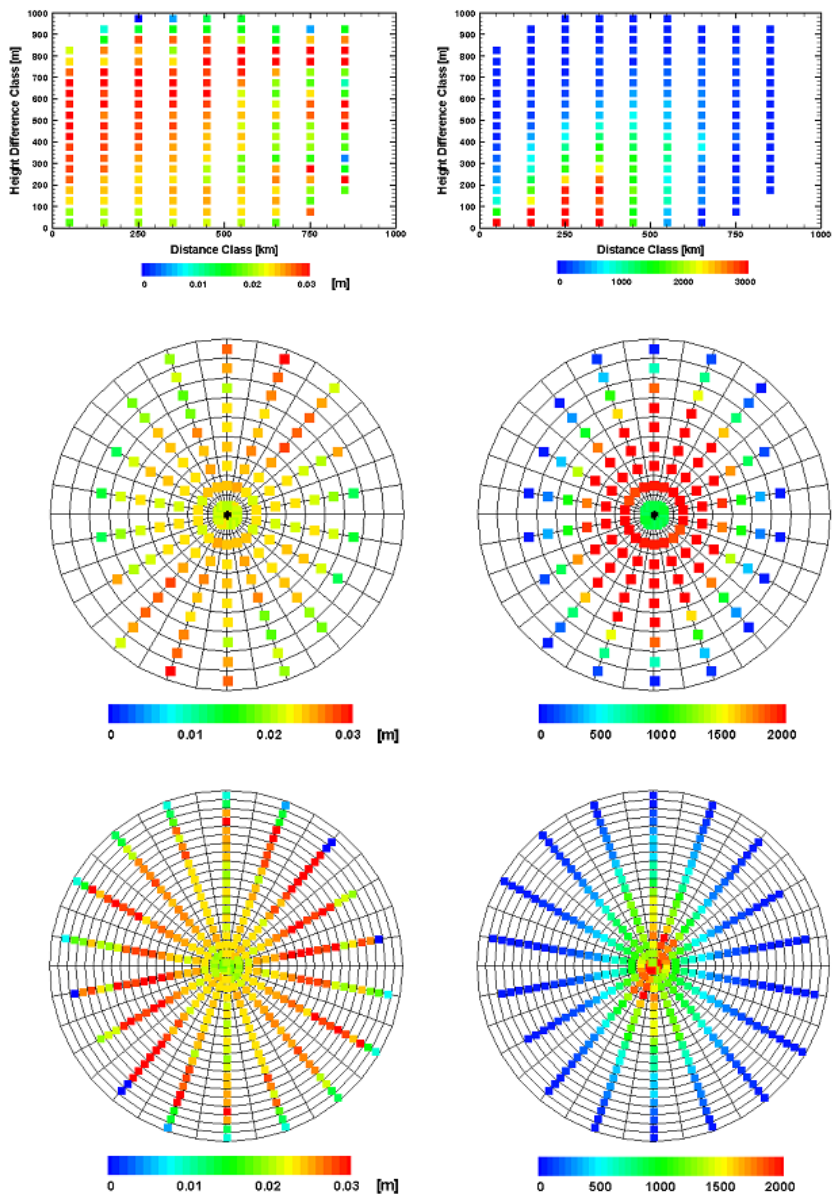

Fig. 6. RMS of geoid slope differences for the EGM2008 and GOCE-OGMOC high resolution models with full resolution (degree 2190) and GNSS-levelling points in Germany (data set G07). The RMS value is computed and shown for the combination of two classes. 1. Row: Distance and height differences, 2. Row: Distance and orientation (circles represent distance classes in steps of $100 \mathrm{~km}$ ), 3. Row: Height differences and orientation (circles represent height difference classes in steps of $50 \mathrm{~m}$ ).

problems which need to be considered for future developments. The following detailed conclusions are summarized from the results.

1. The long wavelengths (e.g. up to degree 60) are mostly determined from GRACE satellite data. Therefore, a GRACE model shall serve as the fundamental basis for any high resolution gravity field.

2. The medium wavelengths up to degree 200 are dominated by the GOCE satellite data. GOCE data are responsible for about $80 \%$ of the differences to the EGM2008 model. As found by the GNSS-levelling comparisons, this represents real improvements in the global models.
3. New and improved surface and altimetric gravity data are responsible for about $20 \%$ of the differences of the new models to EGM2008 in a global average. These data dominate the differences in the spectral range from degree 200 to 500 . In a global average only a minor contribution of the improved data is visible in the very short wavelengths above degree 500 . But, for local comparisons improved surface data play an important role, as it is shown by the GNSS-levelling comparisons in Brazil.

4. GNSS-levelling geoid heights suffer from systematic problems mostly caused by the spirit levelling. Therefore, when comparing geoid heights from global mod- 
RMS geoid slope differences EGM2008
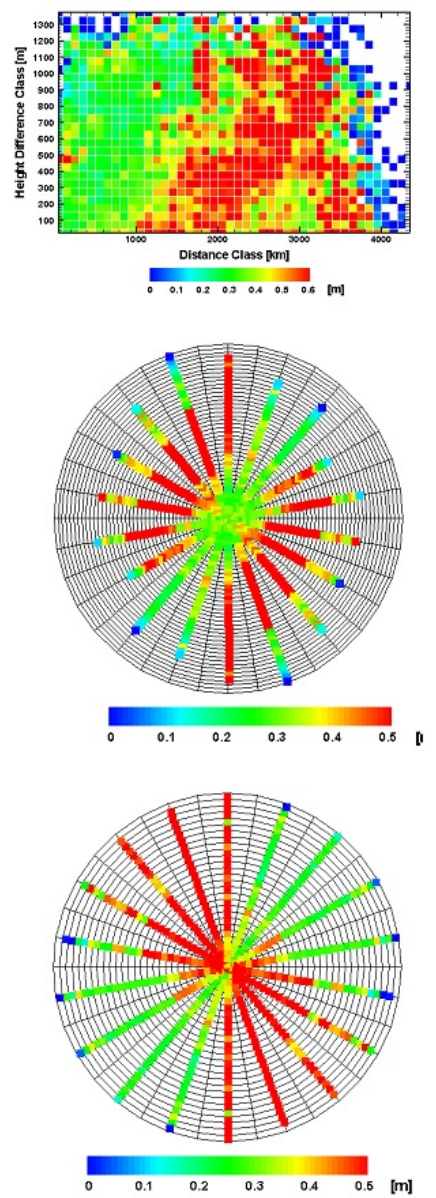

RMS geoid slope differences GOCEOGMOC
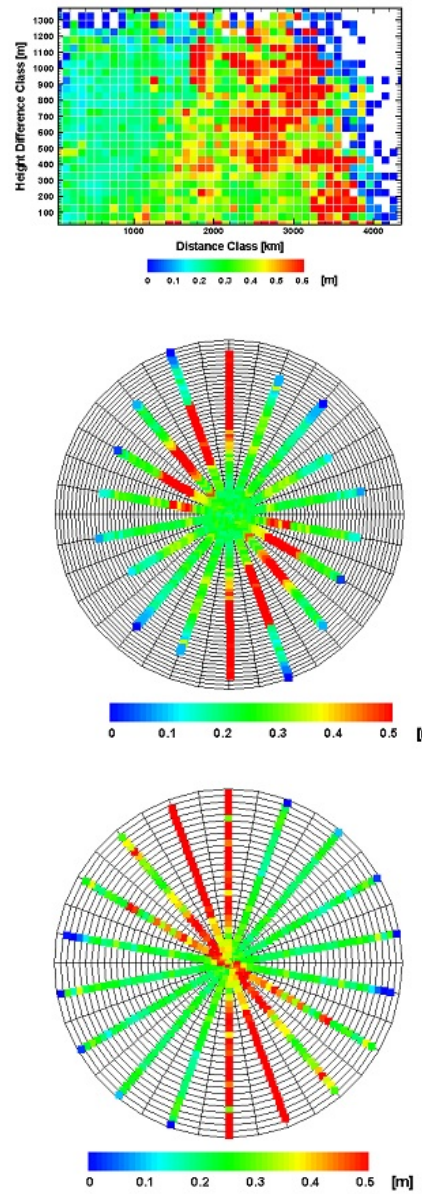

Number of slope differences for class combination
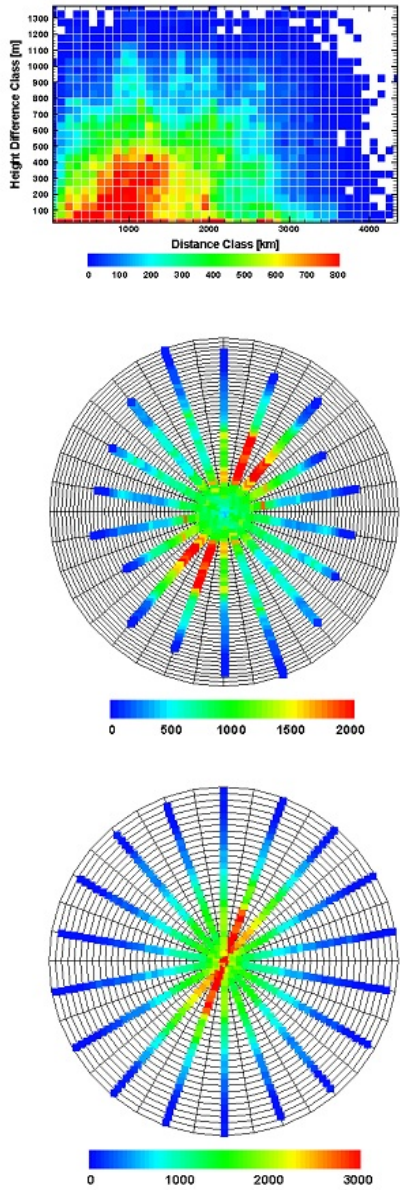

Fig. 7. RMS of geoid slope differences for the EGM2008 and GOCE-OGMOC high resolution models with full resolution (degree 2190) and GNSS-levelling points in Brazil (data set G02). The RMS value is computed and shown for the combination of two classes. 1. Row: Distance and height differences, 2. Row: Distance and orientation (circles represent distance classes in steps of $100 \mathrm{~km}$ ), 3. Row: Height differences and orientation (circles represent height differenceclasses in steps of $50 \mathrm{~m}$ ).

els to GNSS-levelling geoid heights a correction surface needs to be applied. In this analysis for each regional comparison a planar correction surface is estimated and applied. In principle higher order correction surfaces could be considered, but one has to take care not to absorb possible systematic problems from the GNSS observations or the global high resolution gravity field models. Therefore, we regard a planar correction surface as best approximation in this case.

5. Three classes of models can be identified when comparing GNSS-levelling geoid heights for the available regional data sets with geoid heights computed from the high resolution models. The EGM2008 model, which doesn't contain any GOCE data performs worst for most regions. The GECO, SGG-UGM-1 and the EIGEN6-C4 (all based on GOCE information) represent the middle class exhibiting partially better and partially worse results compared to the average. The GOCE-OGMOC and the PGM2017, which are based on GRACE, GOCE and improved terrestrial and altimetric gravity information in average perform best.

6. For refining the analysis of geoid differences various degrees of truncation are applied to the high resolution models. Thereby, the remaining omission error is computed from the most recent PGM2017 model (and from a residual topographic gravity model). The statistic of these differences confirm that up to degree 200 (may be 220) all models, which are using GOCE 
(and GRACE) information, perform significantly better than EGM2008. For the Brazilian data set it is shown that about $80 \%$ of the overall improvement can be attributed to the inclusion of GOCE data, while the remaining improvements are caused by better surface gravity data.

7. For the German data set the contribution of the GOCE information is visible as well, but for the higher degrees, which are determined by the terrestrial gravity data, the PGM2017 model performs worst. This is unexpected and requires additional analyses as one should expect that at least the level of improvement from the GOCE data is kept for the full resolution of the model as well.

8. Geoid slope differences between the global models and the GNSS-levelling data are computed and statistically analysed. RMS values of differences are computed for distance, height difference and orientation classes. Results confirm the findings from the pure height differences to a large extent, but also exhibit some new features. Geoid slope differences increase for larger distances and larger height differences, which can be attributed to spirit levelling errors (not taken out by the planar correction surface). Geoid slope differences with respect to their orientation show varying dominant angles for different regional data sets. This means that larger errors exist in the geoid slopes at specific directions, which most likely can be attributed to the spirit levelling. But it also can't be excluded that other error sources are entering there as well. This needs further studies in order to gain a complete understanding of this process.

9. Geoid slope differences are further analysed by performing statistics for combinations of distance, height difference and orientation classes. In general, the results confirm the previous conclusions, but offer in addition the possibility to get a closer look into problematic areas. This tool mostly is applicable to identify problems in GNSS-levelling observations.

Summarizing all results one can state that in areas with good ground data coverage one can assume that the most recent high resolution global gravity field models reach a quality of $1.5 \mathrm{~cm}$ or better. This is shown by comparisons of model geoid heights with the high quality GNSS-levelling data set for Germany, where we reach RMS values of geoid height differences (after subtracting a planar correction surface) of 1.5 to $2.1 \mathrm{~cm}$. This error is composed by spirit levelling error, GNSS height error, commission error of the global models and residual omission error. It is hardly possible to disentangle the total error into its individual components, but in the case of Germany one can assume that at least $0.5 \mathrm{~cm}$ of the total error is from other sources than the global model. Consequently, a $1 \mathrm{~cm}$ geoid from the global model seems to be feasible. For areas with less good ground data coverage the situation is different, but already with the satellite contribution the total error is reduced drastically.

Acknowledgements: The authors are grateful to "The Helmholtz Centre Potsdam German Research Centre for Geosciences - GFZ” for operating "The International Centre for Global Earth Models (ICGEM)" as a service of the "International Association of Geodesy".

The authors are grateful to the institutions who made available the GNSS-levelling data as shown in Table 2.

\section{References}

Andersen O.B. and Knudsen P., 2009, The DNSC08 mean sea surface and mean dynamic topography. J. Geophys. Res., 114, C11, https://doi.org/10.1029/2008JC005179.

Barthelmes F. and Köhler W., 2016, International Centre for Global Earth Models (ICGEM). In H. Drewes, F. Kuglitsch, J. Adám et al., The Geodesists Handbook 2016, J Geod, 90, 10, 907-1205, https://doi.org/10.1007/s00190-016-0948-z

Bingham R.J., Haines K. and Hughes C.W., 2008, Calculating the Ocean's Mean Dynamic Topography from a Mean Sea Surface and a Geoid, J Atm Ocean Tech 25, 10, 1808-1822 https://doi. org/10.1175/2008JTECH0568.1

Brockmann J.M., Zehentner N., Höck E., Pail R., Loth I., Mayer-Gürr T. and Schuh W.D., 2014, EGM_TIM_RL05: An independent geoid with centimeter accuracy purely based on the GOCE mission, Geophys Res Lett, 41, 22, 8089-8099, https://doi.org/10.1002/ 2014gl061904

Bruinsma S.L., Förste C., Abrikosov O., Marty J.C., Rio M.H., Mulet S. and Bonvalot S., 2013, The new ESA satellite-only gravity field model via the direct approach, Geophys Res Lett, 40, 14, 3607-3612, https://doi.org/10.1002/grl.50716

Evariste N.H., Genyou L., Tabod T.C., Joseph K., Severin N., Alain T. and Xiaoping K.E., 2014, Crustal structure beneath Cameroon from EGM2008, 2014, Geodesy and Geodynamics, 5, 1, 1-10, ISSN 1674-9847, https://doi.org/10.3724/SP.J.1246.2014. 01001.

Förste C., Bruinsma S.L., Abrikosov O., Lemoine J.M., Marty J.C., Flechtner F., Balmino G., Barthelmes F. and R. Biancale, 2014, EIGEN-6C4 The latest combined global gravity field model including GOCE data up to degree and order 2190 of GFZ Potsdam and GRGS Toulouse. GFZ Data Services, http://doi.org/10.5880/icgem.2015.1

Gilardoni M., Reguzzoni M. and Sampietro D., 2016, GECO: a global gravity model by locally combining GOCE data and EGM2008, Stud Geophys Geod, 60, 228, https://doi.org/10.1007/s11200015-1114-4

Gruber T. and Fecher T., 2018, Optimal Ocean Geoid as Reference Surface for Mean Ocean Circulation and Height Systems, Geo- 
physical Research Abstracts, Vol. 20, EGU2018-8691, EGU General Assembly 2018

Gruber T., Rummel R., Abrikosov O. and van Hees R., 2014, GOCE Level 2 Product Data Handbook, Issue 5, European Space Agency, https://earth.esa.int/documents/10174/1650485/ GOCE_Product_Data_Handbook_Level-2

Gruber T., Visser P.N.A.M., Ackermann C. and Hosse M., 2011, Validation of GOCE gravity field models by means of orbit residuals and geoid comparisons. J Geod, 85, 845-860. https://doi.org/10.1007/s00190-011-0486-7.

Heiskanen W.A. and Moritz H., 1967, Physical Geodesy. W.H. Freeman \& Co Ltd, New York

Hirt C., Kuhn M., Claessens S., Pail R., Seitz K. and Gruber T., 2014, Study of the Earth's short-scale gravity field using the ERTM2160 gravity model. Computers \& Geosciences 73, 71-80, https://doi.org/10.1016/j.cageo.2014.09.001

Li J., Shen W.B. and Zhou X., 2015, Direct regional quasi-geoid determination using EGM2008 and DEM: A case study for Mainland China and its vicinity areas, Geodesy and Geodynamics, 6, 6, 437-443, ISSN 1674-9847, https://doi.org/10.1016/j.geog. 2015.12.002

Liang W., 2018, SGG-UGM-1: the high resolution gravity field model based on the EGM2008 derived gravity anomalies and the SGG and SST data of GOCE satellite. GFZ Data Services. http: //doi.org/10.5880/icgem.2018.001
Mayer-Gürr T. and the GOCO Team, 2015, The combined satellite gravity field model GOCO05s, Geophysical Research Abstracts, Vol. 17, EGU2015-12364, EGU General Assembly 2015

NGA (National Geospatial Agency), 2018, The preliminary Earth Gravity Field Model 2017, personal communication

Pail R., Fecher T., Barnes D., Factor J.F., Holmes S.A., Gruber T. and Zingerle P., 2018, Short note: the experimental geopotential model XGM2016, J Geod, 92, 4, 443-451. https://doi.org/10. 1007/s00190-017-1070-6

Pavlis H.K., Holmes S.A., Kenyon S.C. and Factor J.K., 2012, The Development and evaluation of the Earth Gravitational Model 2008 (EGM2008), J. Geophys. Res., 117, B04406, https://doi. org/10.1029/2011JB008916

Rapp R.H., 1997, Use of potential coefficient models for geoid undulation determinations using a spherical harmonic representation of the height anomaly/geoid undulation difference, J Geod 71, 282-289, https://doi.org/10.1007/s001900050096

Sjöberg L.E., 2018, On the topographic bias and density distribution in modelling the geoid and orthometric heights, J Geod Sci, 8, 30-33. https://doi.org/10.1515/jogs-2018-0004

Wang Y.M., Saleh J., Li X. and Roman D.R., 2012, The US Gravimetric Geoid of 2009 (USGG2009): model development and evaluation, J Geod, 86: 165, https://doi.org/10.1007/s00190-0110506-7 